\title{
Surface and Bulk Crystallization of Amorphous Solid Water Films: Confirmation of "Top-Down" Crystallization
}

Chunqing Yuan, R. Scott Smith,* and Bruce D. Kay*

Physical Sciences Division, Pacific Northwest National Laboratory, Richland, Washington 99352

\begin{abstract}
The crystallization kinetics of nanoscale amorphous solid water (ASW) films are investigated using temperature-programmed desorption (TPD) and reflection absorption infrared spectroscopy (RAIRS). TPD measurements are used to probe surface crystallization and RAIRS measurements are used to probe bulk crystallization. Isothermal TPD results show that surface crystallization is independent of the film thickness (from 100 to $1000 \mathrm{ML}$ ). Conversely, the RAIRS measurements show that the bulk crystallization time increases linearly with increasing film thickness. These results suggest that nucleation and crystallization begin at the ASW/vacuum interface and then the crystallization growth front propagates linearly into the bulk. This mechanism was confirmed by selective placement of an isotopic layer $\left(5 \% \mathrm{D}_{2} \mathrm{O}\right.$ in $\left.\mathrm{H}_{2} \mathrm{O}\right)$ at various positions in an $\mathrm{ASW}\left(\mathrm{H}_{2} \mathrm{O}\right)$ film. In this case, the closer the isotopic layer was to the vacuum interface, the earlier the isotopic layer crystallized. These experiments provide direct evidence to confirm that ASW crystallization in vacuum proceeds by a "top-down" crystallization mechanism.
\end{abstract}

*Corresponding Authors 


\section{Introduction}

Amorphous solid water (ASW) is a metastable glassy phase form of water that can be created in the laboratory by vapor deposition onto a cold substrate $(\mathrm{T}<130 \mathrm{~K})$. The properties of ASW are of interest for a variety of reasons including its use as a model for liquid and supercooled liquid water [1-5], its use as a model for studying the properties of amorphous solids, and because it is believed to be the predominant form of water in astrophysical and planetary environments [6-8]. The crystallization of ASW films has been studied using a variety of methods including temperature programmed desorption (TPD) [9-15], adsorbate physisorption [16-20], electron diffraction [21, 22], and infrared techniques [14, 15, 20, 23-25]. Most of these studies either report or assume that ASW crystallization proceeds via a random bulk nucleation and growth mechanism. However, one study reports that crystallization may start at the vacuum interface, although detailed kinetic modeling was required to separate the surface and bulk crystallization rates in the relatively thin film (45 ML) [20, 26].

It has been shown previously that inert adsorbates trapped underneath or within ASW films desorb in an episodic release that occurs in concert with ASW crystallization, a phenomenon called the "molecular volcano" [27, 28]. The abrupt desorption of gases is due to cracks that form during crystallization of the ASW overlayer. Some more recent work studying the crack formation that accompanies ASW crystallization in thicker films showed that crack formation begins at the ASW/vacuum interface [29-31]. In that work, selective placement of the inert gas layer in the ASW film showed that cracks form near the top of the film and propagate downward into the film. Given the link between crystallization and crack formation, these results suggested that ASW crystallization may also begin at the ASW/vacuum interface and proceed into the bulk. 
In the present paper we provide direct evidence that ASW crystallization proceeds via a "topdown" mechanism. This is accomplished by simultaneously monitoring crystallization on the surface and in the bulk as a function of ASW film thickness (100 to $1000 \mathrm{ML}$ ). Temperatureprogrammed desorption (TPD) measurements are used to probe surface crystallization and RAIRS measurements are used to probe bulk crystallization. Our experimental observations are consistent with a two-step model in which nucleation and crystallization begin at the vacuumASW and then the crystallization front propagates into the film bulk.

\section{Material and methods}

The experiments were performed in an ultra-high vacuum system (UHV) with a base pressure of $<10^{-10}$ Torr that has been described in detail previously $[14,32]$. Briefly, a $1 \mathrm{~cm}$ diameter $\operatorname{Pt}(111)$ substrate, cooled by a closed cycle helium cryostat capable of achieving a base temperature of $\sim 25 \mathrm{~K}$, is used as a substrate. The substrate was resistively heated and the temperature was measured by a K-type thermocouple spot-welded to the back. The temperature was measured with a precision of better than $\pm 0.01 \mathrm{~K}$. The absolute temperature was calibrated using the desorption of multilayer $\mathrm{H}_{2} \mathrm{O}$ TPD and estimated to have an accuracy of $\pm 2 \mathrm{~K}$. The Pt substrate was cleaned by $\mathrm{Ne}^{+}$ion sputtering at $1.5 \mathrm{kV}$ and then annealed at $1100 \mathrm{~K}$ in UHV. The Pt substrate was then heated to $1100 \mathrm{~K}$ in the presence of decane to form a single layer of graphene on its surface [33]. The ASW films in this experiment were deposited on top of $50 \mathrm{ML}$ of decane to eliminate (or at least minimize) the potential effects of substrate-induced nucleation. We hypothesize that the long chain hydrocarbon deposited at low temperature will not form an extended ordered surface that could act as a crystallization template. Graphene is inert to 
hydrocarbon decomposition ( $\mathrm{Pt}$ is not) and this provided a clean reproducible surface for growing the ASW/decane films.

Water and decane were deposited using a quasi-effusive molecular beam at normal incidence and at $\sim 30 \mathrm{~K}$. Surface crystallization was measured by monitoring the desorption rates of $\mathrm{H}_{2} \mathrm{O}$ $(\mathrm{m} / \mathrm{z}=18)$ utilizing an Extrel quadrapole mass spectrometer in a line-of-sight configuration. Simultaneous with the desorption measurements, the RAIRS spectra were recorded with a Bruker Vertex 70 Fourier transform infrared spectrometer where the infrared beam was incident on the sample at an angle of $82 \pm 1^{\circ}$. The infrared spectra were acquired with a resolution of $8 \mathrm{~cm}^{-1}$. During a typical isothermal crystallization experiment, 100 infrared spectra were acquired in $900 \mathrm{~s}$, and each spectrum was averaged for 64 scans. To clearly measure the ASW crystallization kinetics by infrared spectroscopy, a $5 \% \mathrm{D}_{2} \mathrm{O}$ in $\mathrm{H}_{2} \mathrm{O}$ liquid solution was used to create the ASW films. In solution, H-D exchange results in the solution being 10\% HOD. The deposited ASW films have an O-D stretching peak near $2450 \mathrm{~cm}^{-1}$ which transforms into a sharp peak at $2426 \mathrm{~cm}^{-1}$ when crystallized. These peaks (compared to the $\mathrm{OH}$ stretch in pure $\mathrm{H}_{2} \mathrm{O}$ ) are sharper because the O-D stretch of HOD is decoupled from interactions with neighboring $\mathrm{OH}$ bonds [34]. The use of the $5 \% \mathrm{D}_{2} \mathrm{O}$ in $\mathrm{H}_{2} \mathrm{O}$ solution facilitated the analysis of the infrared spectra and also allowed for the isotope layer experiments described in section 3.4.

\section{Results and Discussion}

\subsection{Surface Crystallization of ASW Films}

The surface crystallization of ASW films in vacuum was determined using isothermal desorption measurements. All ASW films were deposited on top of $50 \mathrm{ML}$ of decane. The composite 
ASW/decane films were deposited at normal incidence and at $30 \mathrm{~K}$. After deposition the films were heated via a linear temperature ramp $(1 \mathrm{~K} / \mathrm{s})$ to and then held at $150 \mathrm{~K}$. Figure 1 (a) displays the isothermal TPD spectra for a series of ASW films with thicknesses of 100 (blue curve), 200 (red curve), 500 (black curve), and $1000 \mathrm{ML}$ (green curve). The vertical dashed line $(t=0)$ marks the time when the temperature reaches $150 \mathrm{~K}$. The desorption spectra for all four ASW thicknesses are nearly identical and have the same desorption rate versus time dependence that has been observed previously $[11,15]$. This desorption behavior is due to the metastable amorphous phase having a higher free energy than the more stable crystalline phase. The higher free energy means that the amorphous phase has a higher vapor pressure than the crystalline phase and thus a higher desorption rate. The isothermal spectra in Fig. 1(a) show that upon reaching $150 \mathrm{~K}$ the desorption rate is at its maximum value. Subsequently the rate decreases in time until about $200 \mathrm{~s}$ where the rate plateaus. This is because the initial desorption rate is from a film that is nearly all amorphous but as the surface begins to crystallize, the rate decreases until the film's surface is entirely crystalline.

The crystallization kinetics can be obtained by analyzing the time dependence of the isothermal desorption rate. In the analysis, the initial desorption rate ( $\sim \mathrm{s}$ in Figure 1(a)) is taken to be the desorption rate from a surface that is $100 \%$ amorphous, Rate $_{A S W}$, and the desorption rate in the plateau region ( $200 \mathrm{~s})$ is taken to be desorption rate from a surface that is $100 \%$ crystalline, Rate $_{C I}$. The desorption rate in the intermediate region is linear combination of the two desorption rates,

$$
\text { Rate }=\text { Rate }_{A S W} \cdot(1-x(t))+\text { Rate }_{C I} \cdot x(t)
$$

where $x(t)$ is the fraction crystallized at the surface. The fraction crystallized versus time, $x(t)$, is 
determined using the lever-rule type construction in the equation:

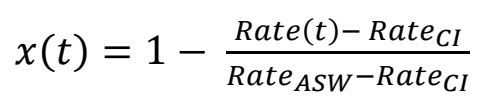

Figure 1(b) displays the $x(t)$ curves obtained from the isothermal TPD spectra in Figure 1(a) for ASW thicknesses of 100 (blue curve), 200 (red curve), 500 (black curve), and 1000 ML (green curve). The curves for all thicknesses are similar in shape and nearly aligned as is expected since the desorption spectra are nearly identical. The results in Figure 1 confirm that the surface crystallization kinetics of ASW films in vacuum are thickness independent from 100 to 1000 ML.

The observed thickness independent surface crystallization kinetics could be explained by two possible mechanisms. The first mechanism is one where there are random nucleation sites evenly distributed throughout the film. In this case, no matter the film thickness, the surface would crystallize at the same rate given that there is an equal probability of a nearby nucleation site. In this mechanism the bulk crystallization kinetics would also be independent of thickness. The second mechanism is one where there is a preference for nucleation at the ASW surface/vacuum interface. In this case, if the surface is the same for all film thicknesses, the surface crystallization rate would be the same. However, in this mechanism the bulk crystallization kinetics could be quite different than those on the surface. To distinguish between these two possible mechanisms, in the next section we measure the bulk crystallization kinetics using RAIRS.

\subsection{Bulk Crystallization of ASW Films}

The bulk crystallization kinetics of ASW films $\left(5 \% \mathrm{D}_{2} \mathrm{O}\right.$ in $\left.\mathrm{H}_{2} \mathrm{O}\right)$ were determined using RAIRS 
spectra obtained simultaneously with the isothermal desorption experiments in Figure 1. Figure 2 displays a time series of RAIRS spectra of the O-D stretch region of HDO for the 100 ML thick ASW film experiment in Figure 1. The O-D stretching band for a 100\% amorphous film (red line) is a relatively broad peak and is centered at $2450 \mathrm{~cm}^{-1}$. During crystallization the peak shifts to lower frequency $\left(2426 \mathrm{~cm}^{-1}\right)$, sharpens, and increases in intensity. The blue curve is the spectrum from a $100 \%$ crystalline film. The time series of spectra have a clear isosbestic point, which suggests that the intermediate spectra are a combination of the pure ASW and crystalline ice spectra. Therefore we can take a cut at the crystalline peak center, $2426 \mathrm{~cm}^{-1}$ (vertical dashed line) to determine $x(t)$ for the bulk ASW crystallization. A formulation analogous to that in Eq. 2 is used to covert the absorbance data to a normalized fraction crystallized.

Figure 3 displays the fraction crystallized versus time, $x(t)$, obtained from RAIRS experiments analyzed using a cut through a set of time resolved spectra as described in Figure 2. The $x(t)$ curves are for ASW films with thicknesses of 100 (blue curve), 200 (red curve), 500 (black curve), and $1000 \mathrm{ML}$ (green curve). The data were obtained in concert with the $150 \mathrm{~K}$ isothermal desorption experiments in Figure 1. The $x(t)$ curves for all thicknesses display an induction period of about $100 \mathrm{~s}$, but in contrast to the surface crystallization results, the RAIRS data show that the bulk crystallization kinetics are thickness dependent. Specifically, the crystallization half-times, $t_{1 / 2}$, increase linearly with ASW film thickness (as shown in Figure 6 below). The linear thickness dependence of bulk crystallization times suggests that the bulk crystallization process is not occurring uniformly throughout the ASW film. 


\subsection{Comparison of Surface and Bulk Crystallization Kinetics}

Figure 4 displays the combined fraction crystallized versus time, $x(t)$, results from the isothermal desorption experiments in Figure 1 (solid lines) and the RAIRS experiments in Figure 4 (dashed lines). The $x(t)$ curves are for ASW films with thicknesses of 100 (blue curves), 200 (red curves), 500 (black curves), and $1000 \mathrm{ML}$ (green curves). The desorption experiments, which measure surface crystallization, are clearly independent of thickness. In contrast, the RAIRS experiments, which measure bulk crystallization, are clearly thickness dependent. Also note that surface crystallization begins $\sim 20 \mathrm{~s}$ and is mostly complete by $\sim 200 \mathrm{~s}$, whereas the bulk crystallization curves have an induction period of $\sim 80 \mathrm{~s}$ before the onset of crystallization. The combined surface and bulk results suggest a two-step crystallization mechanism. In the first step nucleation and crystallization begin at the ASW/vacuum interface and crystallize the surface. In the second step, a crystalline front propagates into the bulk. This mechanism accounts for both the thickness independence of the surface crystallization and the thickness dependence of the bulk crystallization. While it is plausible to assume that the linear time versus thickness dependence observed for bulk crystallization is due to a crystallization front propagating from the rapidly crystallized ASW-vacuum interface surface into the bulk (top-down), it is possible that another mechanism could account for the RAIRS observations in Fig. 3. For example, a linear dependence of the bulk crystallization time versus thickness could also be explained by a "bottom-up" mechanism. While a "bottom-up" mechanism may not be consistent with the desorption experiment results, we nonetheless wanted to confirm the "top-down" mechanism. In the next section we provide direct evidence for a "top-down" crystallization mechanism. 


\subsection{Confirmation of "Top-Dow" Crystallization Mechanism}

In this section we use the selective placement of an isotopic layer in an $\mathrm{H}_{2} \mathrm{O}$ film to confirm the "top-down" crystallization mechanism. Composite ASW films with a total thickness of $1000 \mathrm{ML}$ were deposited at normal incidence at $30 \mathrm{~K}$ on top of $50 \mathrm{ML}$ of decane. Isothermal RAIRS experiments were conducted at $150 \mathrm{~K}$. The $1000 \mathrm{ML}$ ASW film was comprised of $900 \mathrm{ML}$ of $\mathrm{H}_{2} \mathrm{O}$ and a $100 \mathrm{ML}$ isotopic layer $\left(5 \% \mathrm{D}_{2} \mathrm{O}\right.$ in $\mathrm{H}_{2} \mathrm{O}$ ) placed at various positions in the film. Figure 5 displays $x(t)$ curves from experiments where the $100 \mathrm{ML}$ isotopic layer was placed at the top of the film (blue curve), 300 ML below the top (red curve), 600 ML below the top (black curve), and at the bottom (green curve) of the $1000 \mathrm{ML}$ composite film (see schematic in Figure 5). A cut at $2426 \mathrm{~cm}^{-1}$ was used to determine $x(t)$ and thus the data in Figure 5 are sensitive to the OD stretch in HOD. Experiments using homogenous films doped with 10\% HDO indicate that the crystallization kinetics of $\mathrm{H}_{2} \mathrm{O}$ and $\mathrm{HOD}$ are identical. The results show that the $x(t)$ curves shift to longer time the farther the isotopic layer is from the vacuum surface. Particularly note that the onset times also shift with the isotopic layer's distance from the top of the film. These results suggest that the onset of crystallization does not begin in the isotopic layer until the crystallization front reaches its position in the film.

Figure 6 is a plot of the $t_{1 / 2}$ values versus the midpoint of the film thickness for the results in Figure 3 (blue circles) and Figure 5 (red squares). For the experiments in Figure 3 where the entire film is $5 \% \mathrm{D}_{2} \mathrm{O}$ in $\mathrm{H}_{2} \mathrm{O}$, the midpoint is determined from the entire film thickness. For example, in the 1000 ML experiment in Figure 3 the midpoint is 500 ML. For the experiments in Figure 5 where the isotopic layer is located at different positions in the film, the midpoint is based on its location in the film. For example, in the experiment where the $100 \mathrm{ML}$ isotopic 
layer is $300 \mathrm{ML}$ from the top the midpoint is $350 \mathrm{ML}$. The $t_{1 / 2}$ values from the two separate experiments are closely aligned and can be fit by a common line (dashed curve). This result provides additional evidence that the crystallization front that begins at the vacuum surface of the ASW film propagates at a constant rate into the bulk. The inverse fit line slope is a measure of the growth rate (ML/s) and the intercept minus the time to reach the isothermal temperature is a measure of the induction time. The values here, $3 \mathrm{ML} / \mathrm{s}$ for growth and $120 \mathrm{~s}$ for the induction time, are in good agreement with the growth rate and induction time values for crack formation (Figure 8 of reference [30]). We have previously shown that the surface roughness of ASW films deposited at normal incidence does not depend on film thickness.[35-37] For this reason we do not ascribe the observed thickness dependent crystallization rates to differences in surface roughness.

\section{Conclusion}

By direct measurement of the surface (using TPD) and bulk (using RAIRS) ASW crystallization kinetics as a function of film thickness, we clearly show that the crystallization of ASW films in vacuum proceeds via a "top-down" crystallization mechanism. More specifically, ASW crystallization proceeds via a two-step process. In the first step, nucleation and growth begin at the vacuum interface where the entire surface rapidly crystallizes. In the second step, the surface crystalline layer acts as a template that allows for a crystallization front to rapidly propagate into the bulk. The observed linear time dependence on film thickness suggests that the combined surface nucleation and growth front propagation happen faster than bulk nucleation, at least in films up to $1000 \mathrm{ML}$. For example, if nucleation was occurring at a significant rate in the bulk, 
one would expect to see an increase in the crystallization rate (decrease in the crystallization time) with thickness due to an increase in the sites from where crystalline growth could occur.

The preference for nucleation at the vacuum interface is likely due to the higher mobility that surface molecules have compared to those in the bulk. Note that the effects of enhanced surface mobility may extend several layers into the film. Our results mean that prior work using only surface sensitive techniques, such as desorption [9-15] and adsorbate physisorption [16-20], likely do not provide information relevant to bulk nucleation. Surface sensitive experiments may provide information relevant to processes in the environment or in astrophysical ices but it is the bulk nucleation rate that is relevant to processes in supercooled liquid water. Future work will focus on varying the isothermal temperatures to further quantify the ASW kinetics. Temperature may change the importance of surface nucleation on the overall crystallization kinetics. For example, if the activation energy for bulk nucleation is greater than that for surface nucleation, the relative contribution of bulk nucleation and crystallization will increase at higher temperature. Similarly, bulk nucleation may contribute to the overall crystallization rate in thicker films where the top-down crystallization front takes longer to traverse the entire film. In addition, experiments where the effects of the vacuum-surface nucleation are eliminated will be conducted. For example, ASW films deposited between two hydrocarbon layers should eliminate the surface nucleation and allow for the determination of the "true" bulk nucleation rate. Quantitative information on bulk nucleation and growth crystallization kinetics of ASW films is needed to increase our understanding of liquid and supercooled liquid water.

\section{Acknowledgment}


This work was supported by the U.S. Department of Energy (DOE), Office of Science, Office of Basic Energy Sciences, Division of Chemical Sciences, Geosciences, and Biosciences. The research was performed using EMSL, a national scientific user facility sponsored by DOE's Office of Biological and Environmental Research and located at Pacific Northwest National Laboratory, which is operated by Battelle for the DOE. 


\section{References}

[1] P.G. Debenedetti, Metastable liquids: Concepts and principles, Princeton University Press, Princeton ,N.J., 1996.

[2] P.G. Debenedetti, J. Phys.-Condes. Matter, 15 (2003) R1669-R1726.

[3] C.A. Angell, Annu. Rev. Phys. Chem., 55 (2004) 559-583.

[4] C.A. Angell, Science, 319 (2008) 582-587.

[5] R.S. Smith, N.G. Petrik, G.A. Kimmel, B.D. Kay, Accounts Chem. Res., 45 (2012) 33-42.

[6] M.P. Collings, M.A. Anderson, R. Chen, J.W. Dever, S. Viti, D.A. Williams, M.R.S.

McCoustra, Mon. Not. Roy. Astron. Soc., 354 (2004) 1133-1140.

[7] D.A. Williams, W.A. Brown, S.D. Price, J.M.C. Rawlings, S. Viti, Astron. Geophys., 48 (2007) 25-34.

[8] D.J. Burke, W.A. Brown, Phys. Chem. Chem. Phys., 12 (2010) 5947-5969.

[9] N.J. Sack, R.A. Baragiola, Phys. Rev. B, 48 (1993) 9973-9978.

[10] P. Lofgren, P. Ahlstrom, D.V. Chakarov, J. Lausmaa, B. Kasemo, Surface Science, 367 (1996) L19-L25.

[11] R.S. Smith, C. Huang, E.K.L. Wong, B.D. Kay, Surface Science, 367 (1996) L13-L18.

[12] R.J. Speedy, P.G. Debenedetti, R.S. Smith, C. Huang, B.D. Kay, Journal of Chemical Physics, 105 (1996) 240-244.

[13] P. Lofgren, P. Ahlstrom, J. Lausma, B. Kasemo, D. Chakarov, Langmuir, 19 (2003) 265274.

[14] R.S. Smith, T. Zubkov, B.D. Kay, Journal of Chemical Physics, 124 (2006) 114710. 
[15] R.S. Smith, J. Matthiesen, J. Knox, B.D. Kay, J. Phys. Chem. A, 115 (2011) 5908-5917.

[16] Z. Dohnalek, G.A. Kimmel, R.L. Ciolli, K.P. Stevenson, R.S. Smith, B.D. Kay, Journal of Chemical Physics, 112 (2000) 5932-5941.

[17] D.J. Safarik, C.B. Mullins, Journal of Chemical Physics, 117 (2002) 8110-8123.

[18] D.J. Safarik, R.J. Meyer, C.B. Mullins, Journal of Chemical Physics, 118 (2003) 4660-4671.

[19] D.J. Safarik, C.B. Mullins, Journal of Chemical Physics, 119 (2003) 12510-12524.

[20] E.H.G. Backus, M.L. Grecea, A.W. Kleyn, M. Bonn, Physical Review Letters, 92 (2004) 236101.

[21] P. Jenniskens, D.F. Blake, Science, 265 (1994) 753-756.

[22] P. Jenniskens, D.F. Blake, Astrophys. J., 473 (1996) 1104-1113.

[23] W. Hage, A. Hallbrucker, E. Mayer, G.P. Johari, Journal of Chemical Physics, 100 (1994) 2743-2747.

[24] W. Hage, A. Hallbrucker, E. Mayer, G.P. Johari, Journal of Chemical Physics, 103 (1995) $545-550$.

[25] T. Kondo, H.S. Kato, M. Bonn, M. Kawai, Journal of Chemical Physics, 127 (2007) 094703.

[26] E.H.G. Backus, M. Bonn, Journal of Chemical Physics, 121 (2004) 1038-1049.

[27] P. Ayotte, R.S. Smith, K.P. Stevenson, Z. Dohnalek, G.A. Kimmel, B.D. Kay, Journal of Geophysical Research-Planets, 106 (2001) 33387-33392.

[28] R.S. Smith, C. Huang, E.K.L. Wong, B.D. Kay, Physical Review Letters, 79 (1997) 909912.

[29] R.A. May, R.S. Smith, B.D. Kay, J. Phys. Chem. Lett., 3 (2012) 327-331.

[30] R.A. May, R.S. Smith, B.D. Kay, Journal of Chemical Physics, 138 (2013) 104501. 
[31] R.A. May, R.S. Smith, B.D. Kay, Journal of Chemical Physics, 138 (2013) 104502.

[32] T. Zubkov, R.S. Smith, T.R. Engstrom, B.D. Kay, Journal of Chemical Physics, 127 (2007) 184707.

[33] G.A. Kimmel, J. Matthiesen, M. Baer, C.J. Mundy, N.G. Petrik, R.S. Smith, Z. Dohnalek, B.D. Kay, J. Am. Chem. Soc., 131 (2009) 12838-12844.

[34] M. Fisher, J.P. Devlin, J. Phys. Chem., 99 (1995) 11584-11590.

[35] G.A. Kimmel, Z. Dohnalek, K.P. Stevenson, R.S. Smith, B.D. Kay, Journal of Chemical Physics, 114 (2001) 5295-5303.

[36] G.A. Kimmel, K.P. Stevenson, Z. Dohnalek, R.S. Smith, B.D. Kay, Journal of Chemical Physics, 114 (2001) 5284-5294.

[37] K.P. Stevenson, G.A. Kimmel, Z. Dohnalek, R.S. Smith, B.D. Kay, Science, 283 (1999) 1505-1507. 


\section{Figure Captions}

Figure 1 (a) Isothermal TPD spectra for a series of films with ASW thicknesses of 100 (blue curve), 200 (red curve), 500 (black curve), and 1000 ML (green curve). The ASW films (5\% $\mathrm{D}_{2} \mathrm{O}$ in $\mathrm{H}_{2} \mathrm{O}$ solution) were deposited on top of $50 \mathrm{ML}$ of decane. The composite ASW/decane films were deposited at normal incidence and at $30 \mathrm{~K}$. After deposition the films were heated to and held at $150 \mathrm{~K}$. Time zero (vertical dashed line) marks the time the isothermal temperature is reached. (b) The fraction crystallized versus time, $x(t)$, obtained from the isothermal TPD spectra in (a).

Figure 2 A time series of RAIRS spectra obtained simultaneously during the $150 \mathrm{~K}$ isothermal desorption of the $100 \mathrm{ML}$ thick film Figure 1. Only a subset of the total number of recorded spectra are shown. Displayed is the O-D stretching region for HOD. The red curve is the spectrum from a $100 \%$ amorphous film and the blue curve is the spectrum from a $100 \%$ crystalline film. The time difference between the amorphous and crystalline spectra is about $200 \mathrm{~s}$. The vertical dashed line marks the frequency cut $\left(2426 \mathrm{~cm}^{-1}\right)$ used to determine the bulk crystallization kinetics.

Figure 3 The fraction crystallized versus time, $x(t)$, obtained from RAIRS experiments conducted simultaneously during the $150 \mathrm{~K}$ isothermal desorption experiments in Figure 1 . The time axis is the time elapsed at $150 \mathrm{~K}$ (here and in all subsequent figures). The $x(t)$ curves are for ASW films with thicknesses of 100 (blue curve), 200 (red curve), 500 (black curve), and $1000 \mathrm{ML}$ (green curve). 
Figure 4 A combined plot of the fraction crystallized versus time, $x(t)$, results from the isothermal desorption experiments in Figure 1 (solid lines) and the RAIRS experiments in Figure 4 (dashed lines). The $x(t)$ curves are for ASW films with thicknesses of 100 (blue curves), 200 (red curves), 500 (black curves), and 1000 ML (green curves).

Figure 5 The fraction crystallized versus time, $x(t)$, obtained from RAIRS experiments where a $100 \mathrm{ML}$ isotopic layer $\left(5 \% \mathrm{D}_{2} \mathrm{O}\right.$ in $\left.\mathrm{H}_{2} \mathrm{O}\right)$ of $\mathrm{ASW}$ was placed at various positions in an $\mathrm{H}_{2} \mathrm{O}$ film. The total thickness of the composite film was $1000 \mathrm{ML}$ and it was grown on top of $50 \mathrm{ML}$ of decane. The $100 \mathrm{ML}$ isotopic layer was placed at the top of the film (blue curve), 300 ML below the top (red curve), $600 \mathrm{ML}$ below the top (black curve), and at the bottom (green curve) of the 1000 ML composite film. The experiments were conducted at $150 \mathrm{~K}$.

Figure 6 A plot of the $t_{1 / 2}$ values versus the midpoint of the film thickness for the results in Figure 3 (blue circles) and Figure 5 (red squares). For the data from Figure 3, the midpoint is determined from the entire film thickness. For the data from Figure 5, the midpoint is determined on the location of the $100 \mathrm{ML}$ isotopic layer. The dashed line is a linear fit to the combined $t_{1 / 2}$ data sets. The reciprocal of the slope is $\sim 3 \mathrm{ML} / \mathrm{s}$ and the induction time (yintercept) is $80 \mathrm{~s}$. 
Figure 1
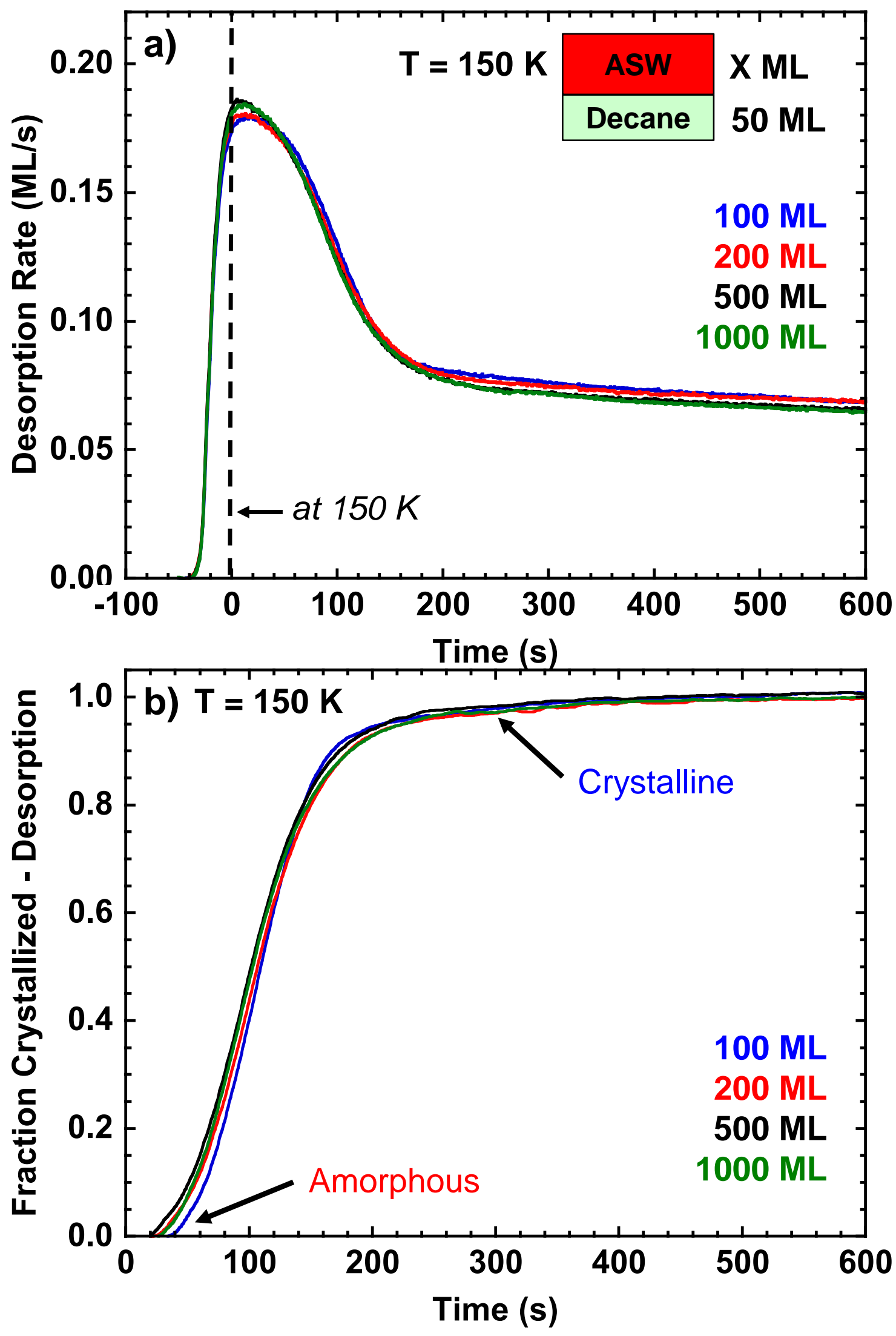
Figure 2

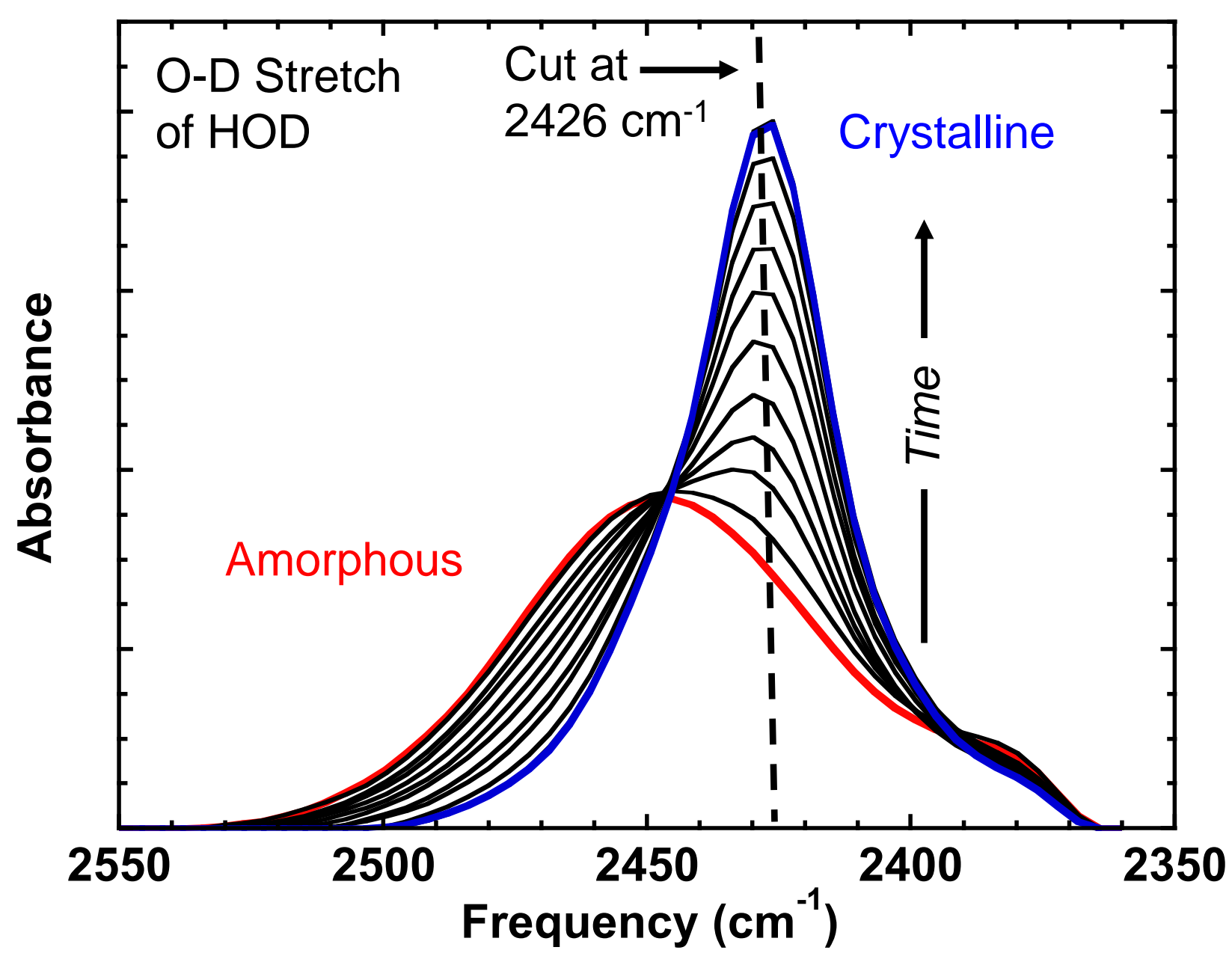


Figure 3

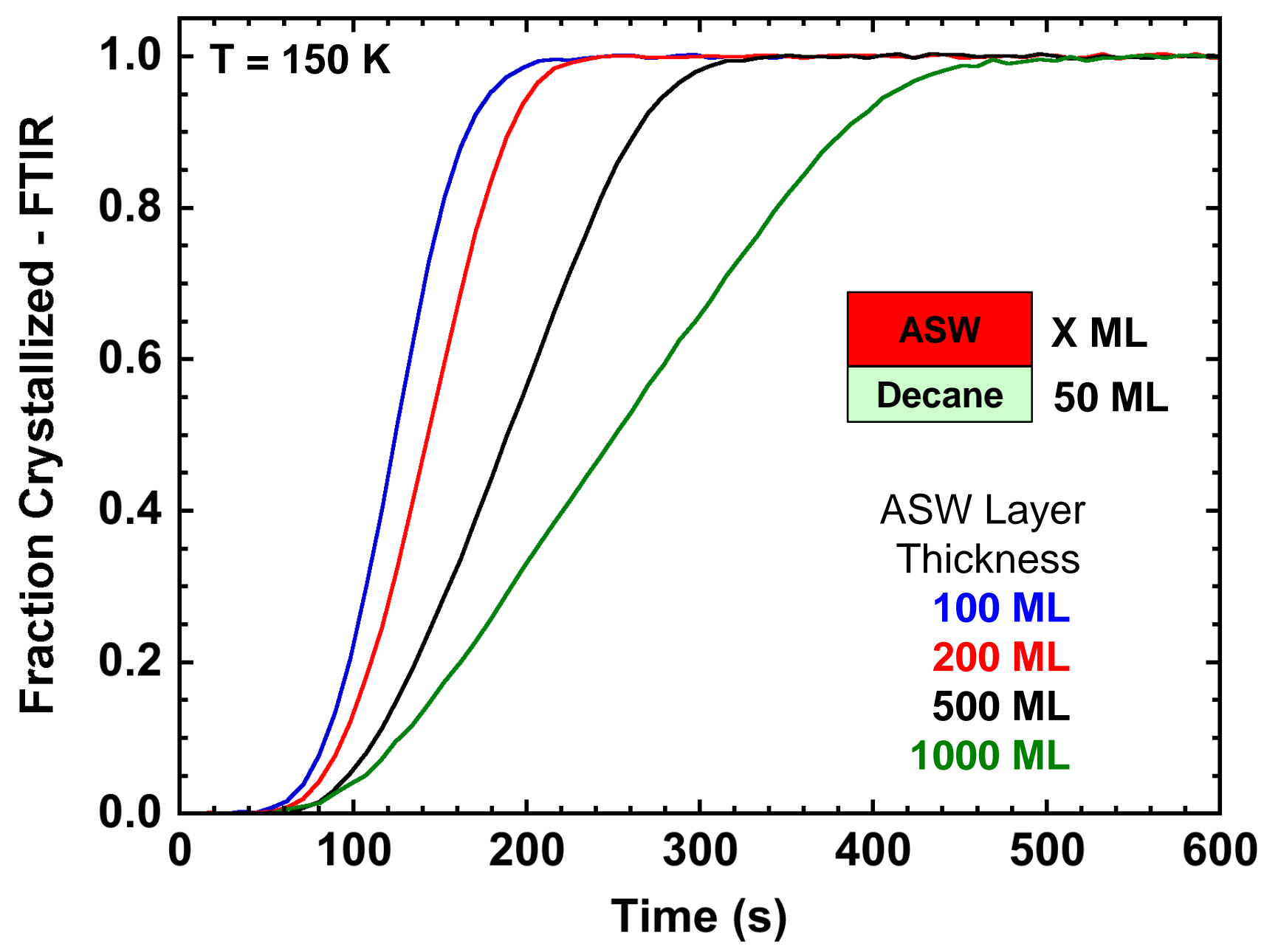


Figure 4

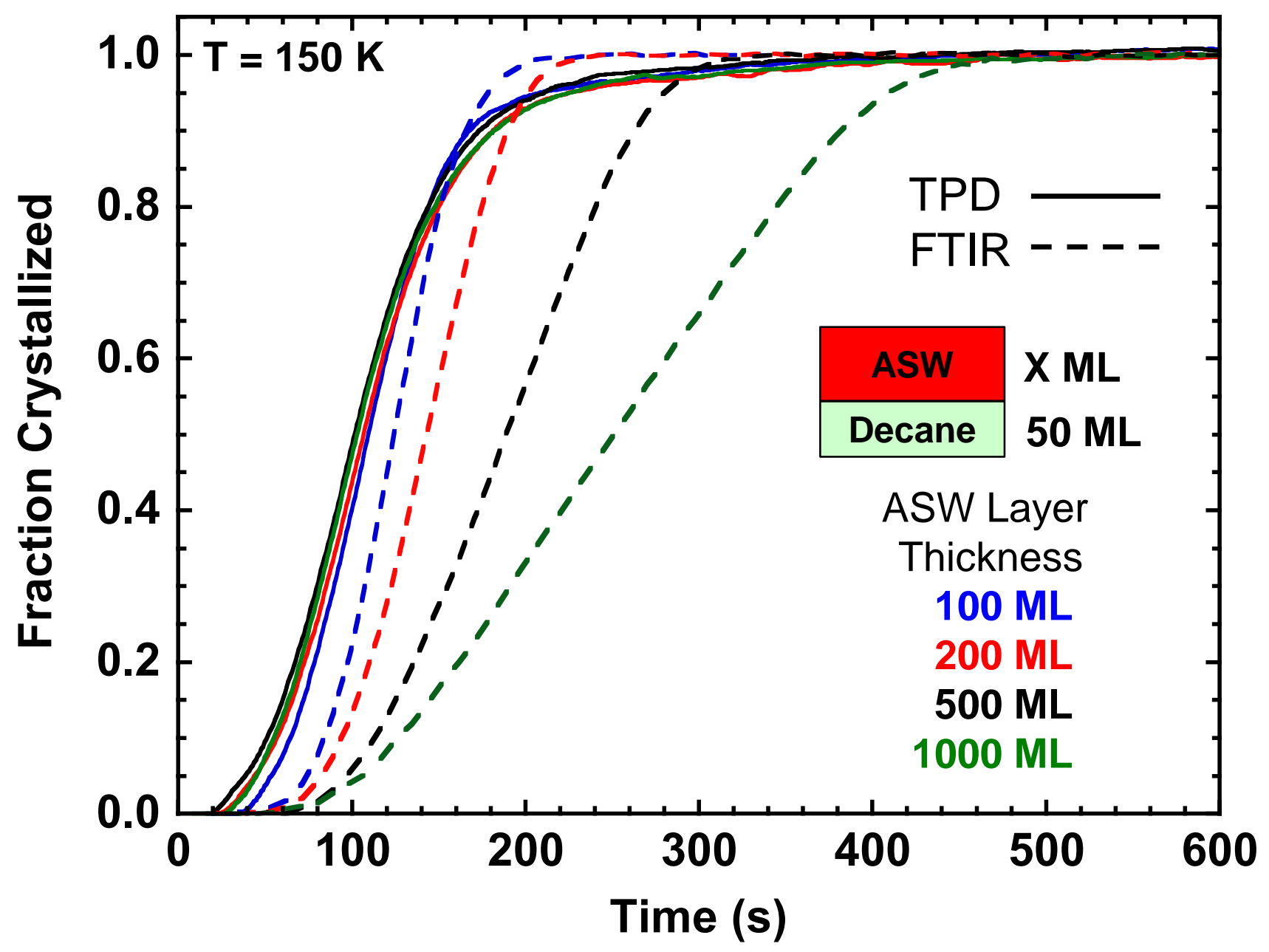


Figure 5

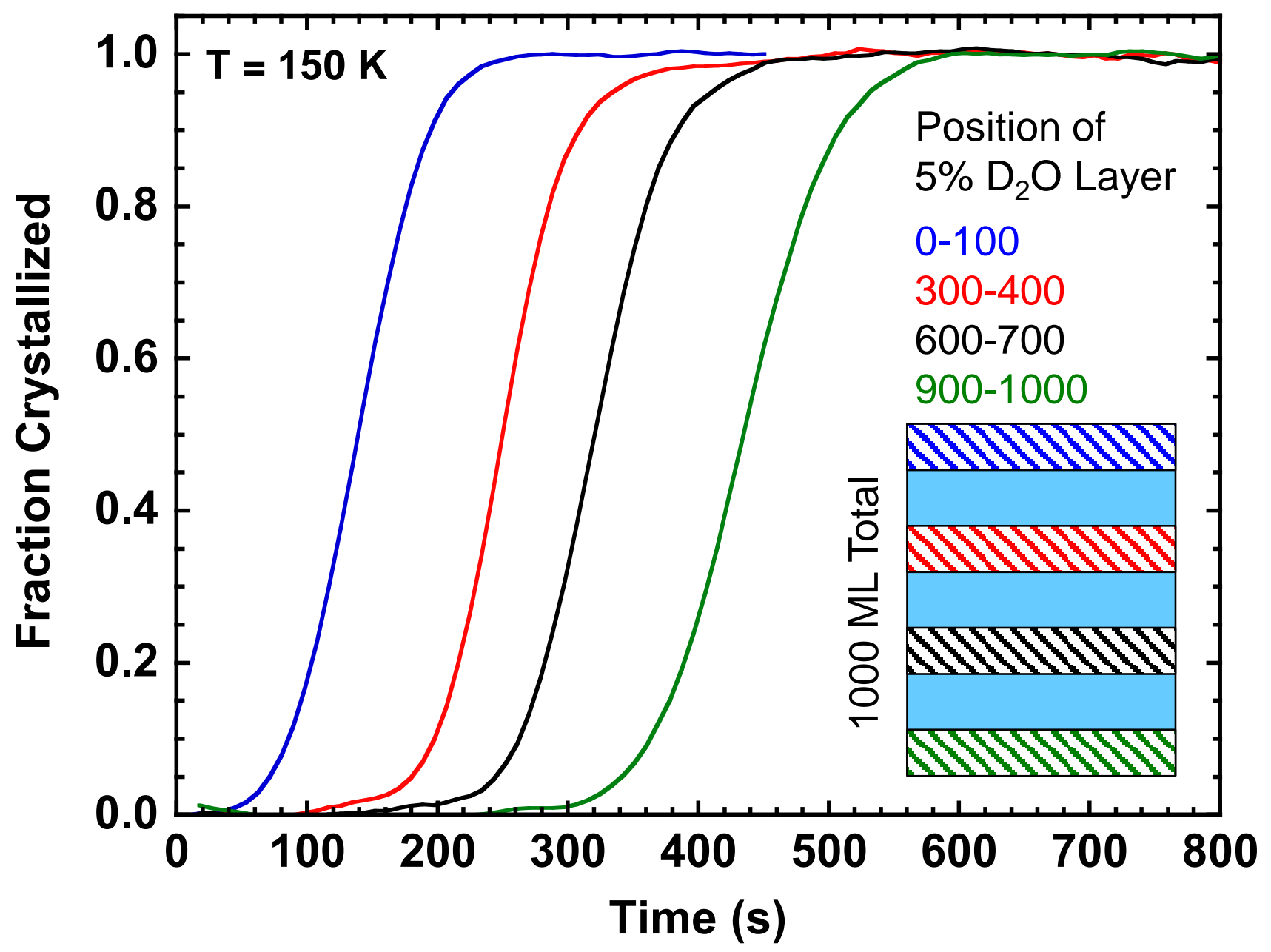


Figure 6

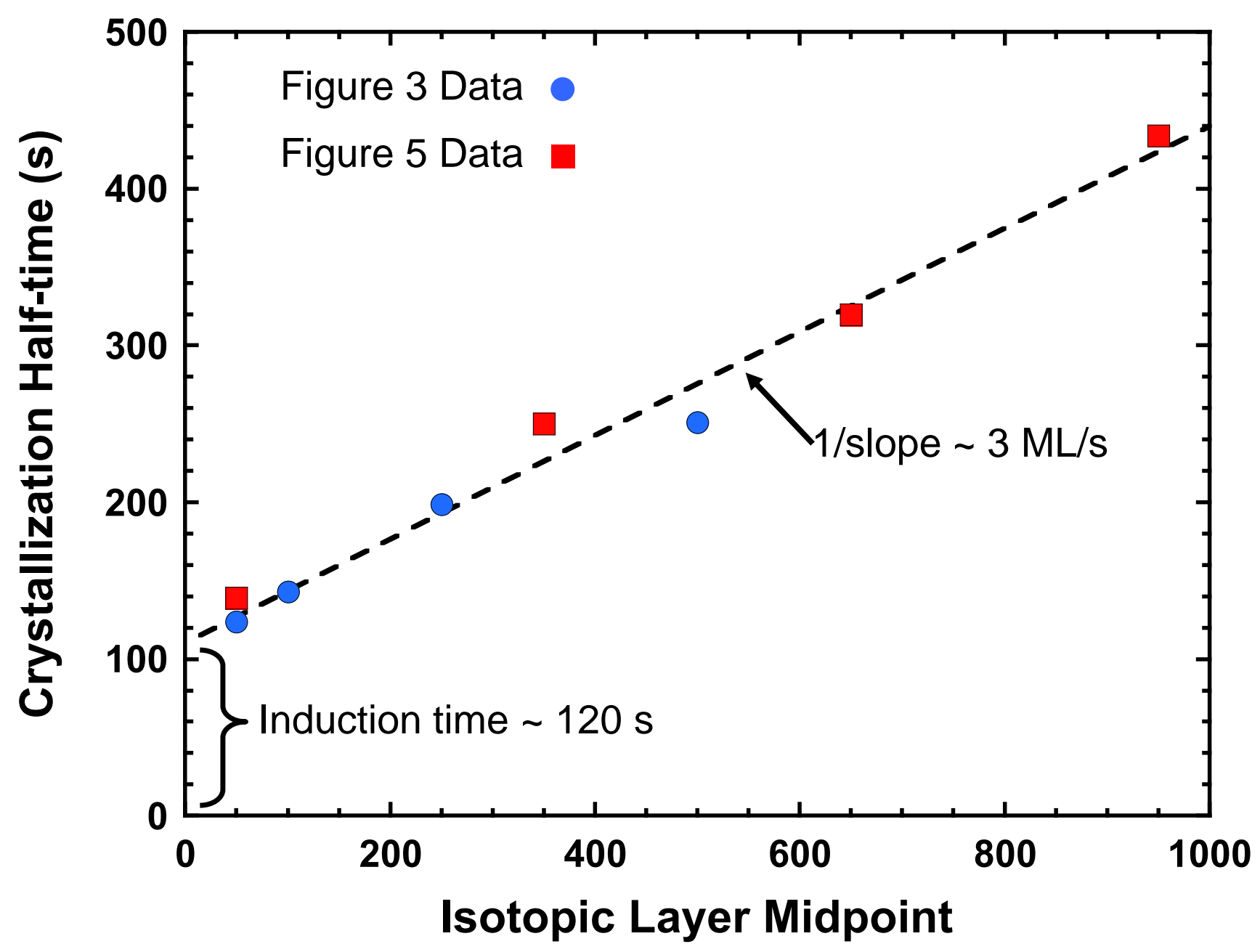


$1000 \mathrm{ML}$

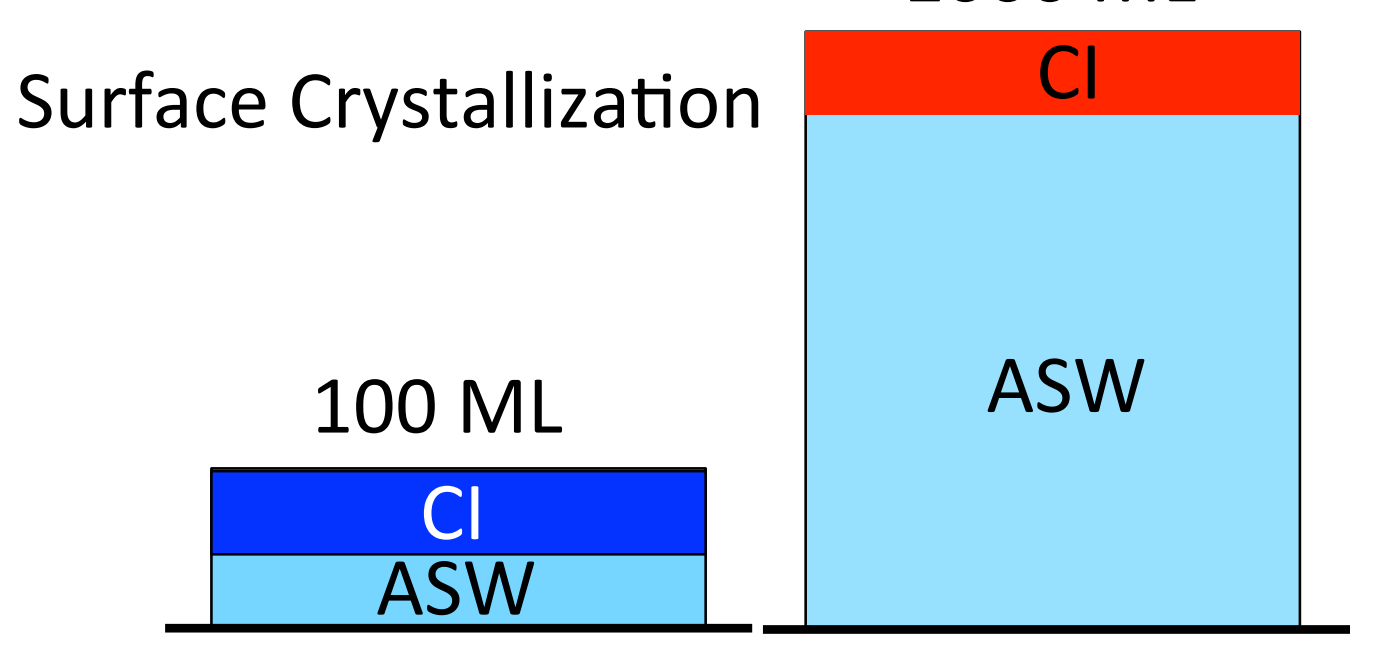

$1000 \mathrm{ML}$

\section{Bulk Crystallization}

$100 \mathrm{ML}$

$$
\mathrm{Cl}
$$
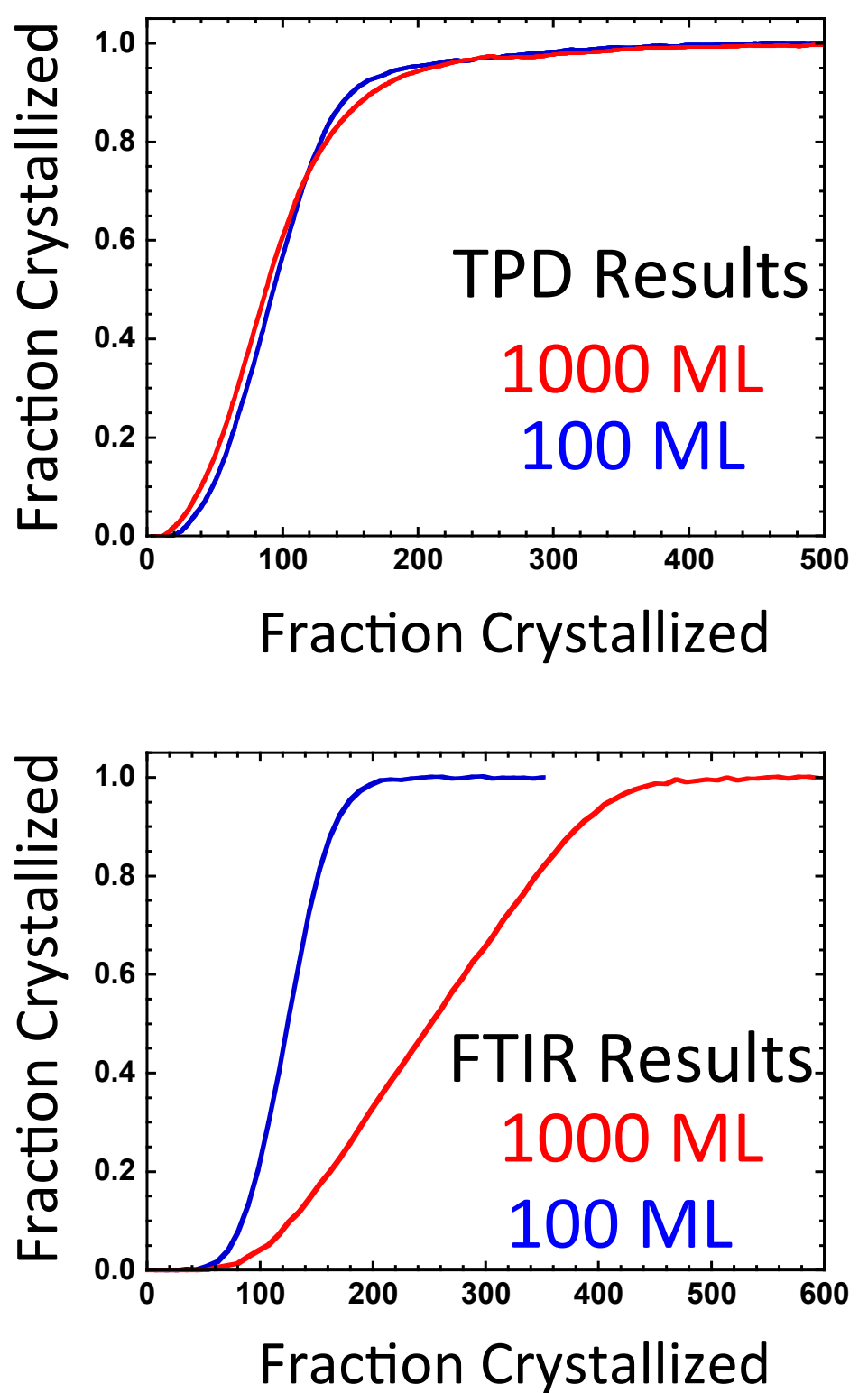